EUROPEAN JOURNAL OF PURE AND APPLIED MATHEMATICS

Vol. 11, No. 1, 2018, 352-361

ISSN 1307-5543 - www.ejpam.com

Published by New York Business Global

\title{
The Proofs of Triangle Inequality Using Binomial Inequalities
}

\author{
Benedict Barnes $^{1, *}$, E. D. J. Owusu-Ansah ${ }^{1}$, S. K. Amponsah ${ }^{1}$, I. A. Adjei ${ }^{1}$ \\ ${ }^{1}$ Department of Mathematics, Kwame Nkrumah University of Science and Technology, \\ Kumasi, Ghana
}

\begin{abstract}
In this paper, we introduce the different ways of proving the triangle inequality $\| u-$ $v\|\leq\| u\|+\| v \|$, in the Hilbert space. Thus, we prove this triangle inequality through the binomial inequality and also, prove it through the Euclidean norm. The first generalized procedure for proving the triangle inequality is feasible for any even positive integer $n$. The second alternative proof of the triangle inequality establishes the Euclidean norm of any two vectors in the Hilbert space.
\end{abstract}

2010 Mathematics Subject Classifications: 44B43, 44B44

Key Words and Phrases: Triangle inequality, triangle through binomial inequality, triangle inequality through Euclidean norm, Hilbert space

\section{Introduction}

The importance of estimating norms cannot be overemphasized on the grounds that, in most practices, the quantification of exact norm of the two vector points in a complete normed space is tedious and sometimes the procedure is cumbersome. In this regard, we fall on the estimation of norms of vector points. Inequalities are used to describe the geometric structures such as boundedness, continuity, uniform non- $l_{1}^{n}$-ness of mappings or operators of the linear spaces and also, the embeddings of one vector space into another vector space. Recently, some applications of the triangle inequality in the operations research have been found, see research papers by $[1,2]$.

The proof of the triangle inequality of the form

$$
\|u+v\| \leq\|u\|+\|v\|,
$$

is well-known and many researchers across the globe have shown different ways for obtaining this result. A lot of studies delineate the origin of inequalities. For example, see

\footnotetext{
${ }^{*}$ Corresponding author.

Email addresses: bbarnes.cos@knust.edu.gh (B. Barnes), degraftt@gmail.com (E. D. J. Owusu-Ansah), skamponsah@knust.edu.gh (S. K. Amponsah) 
a research paper by author in [3]. In [4], the author applied generalized Minkowski's inequality in a generalized vector space to obtain the triangle inequality. The authors in [5], used both the notion of $q$-norm and- $\psi$ norm to construct the triangle inequality. Another version for obtaining this kind of triangle inequality in Banach space was obtained by authors in [6]. The author in [7], obtained an alternative way of proving triangle inequality in Banach space. In [8], the authors applied the $\psi$ - sum of the vector points in the Banach space to construct the triangle inequality. The authors in [9], observed that for any vectors $\left(\mu_{1}, \mu_{2}, \ldots, \mu_{n}\right)$, in the Euclidean space the following inequality holds:

$$
\left\|x_{1}+\ldots, x_{n}\right\|^{p} \leq \frac{\left\|x_{1}\right\|^{p}}{\mu_{1}}+\ldots+\frac{\left\|x_{n}\right\|^{p}}{\mu_{n}} .
$$

The triangle inequality has different versions. One of the particular version which is recent and has attracted much attention of scientists in the $21^{\text {st }}$ century is the triangle inequality of the form:

$$
\|u-v\| \leq\|u\|+\|v\|,
$$

for any two vectors in a normed linear space $V$ [see 10]. This inequality has alternative ways of proving it. For example, see a research paper by authors [11].

In this paper, we present alternative ways of proving triangle inequality in (1); by the use of concept of binomial of two vector points in the Hilbert space. This result generalizes the proof of the triangle inequality. Also, the proof of the triangle inequality is proved through the Euclidean norm. The paper is organized as follows. Section 1 contains the introduction, the new methods for proving the triangle inequality in (1) is seen in section 2 and section (3) contains the summary of this paper.

\section{Main Result}

In this section, we introduce a general procedures for proving the inequality in (1). Firstly, we make use of binomial of two vector points to establish the triangle inequality in the Hilbert space for any positive integer $n$. In addition, we obtain the same result by applying the Young's inequality of the two vector points through the Euclidean norm. These approaches are easier and give better understanding of the triangle inequality.

Definition 1 (Inner product Space). Let $X$ be a vector space of over $K$. A function

$$
(., .): X \times X \rightarrow K
$$

is called an inner product space on the vector space $X$ over $K$ if:

P1: for all $x \in X,(x, x) \geq 0$, and $(x, x)=0$ if and only if $x=0$

P2: $\left(x_{1}+x_{2}, y\right)=\left(x_{1}, y\right)+\left(x_{2}, y\right)$ and $(\alpha x, y)=\alpha(x, y)$, for all $x_{1}, x_{2}, y \in X$ and $\alpha \in K$

P3: $(x, y)=(y, x)$, for all $x, y \in X$. The bar denotes complex conjugate of two vector points in $X($ See, 12)

Thus, an inner product space is a vector space endowed with an inner product. 
Theorem 1 (Cauchy-Schwarz Inequality). If $u$ and $v$ are any two vectors in an inner product space $V$, then

$$
|(u, v)| \leq\|u\|\|v\|
$$

(see, 13).

\subsection{A Proof of Triangle Inequality Through Binomial Inequality}

In this section, we introduce an alternative way of proving the triangle inequality through binomial inequality. By induction, we prove the triangle inequality in (1) as follows. Firstly, we consider an integer $n=2$, we observe the following:

$$
\begin{aligned}
(u+v)^{2} & \geq 0 \\
(u, u)+2(u, v)+(v, v) & \geq 0 \\
\{(u, u)+(v, v)\} & \geq-2(u, v) \\
-\{(u, u)+(v, v)\} & \leq 2(u, v) \\
-\{(u, u)+(v, v)-2(u, v)\} & =4(u, v) \\
-(u-v)^{2} & =4(u, v) \\
\left\|-(u-v)^{2}\right\| & =\|4(u, v)\| \\
\|(u-v)\|^{2} & \leq 4\|u\|\|v\|
\end{aligned}
$$

On other hand, we see that:

$$
\begin{aligned}
(u-v)^{2} & \geq 0 \\
(u, u)-2(u, v)+(v, v) & \geq 0 \\
-2(u, v) & =-\{(u, u)+(v, v)\} \\
2(u, v) & \leq\{(u, u)+(v, v)\} \\
4(u, v) & =\{(u, u)+(v, v)+2(u, v)\} \\
4(u, v) & =(u+v)^{2} \\
\|4(u, v)\| & =\left\|(u+v)^{2}\right\| \\
4\|u\|\|v\| & \leq\|u+v\|^{2}
\end{aligned}
$$

Applying the transitive law to (2) and (3), we obtain

$$
\begin{aligned}
\|u-v\|^{2} & \leq\|u+v\|^{2} \\
\left(\|u-v\|^{2}\right)^{\frac{1}{2}} & =\left(\|u+v\|^{2}\right)^{\frac{1}{2}} \\
\Rightarrow\|u-v\| & =\|u+v\| \\
\Rightarrow\|u-v\| & \leq\|u\|+\|v\|
\end{aligned}
$$

For $n=4$, we observe that:

$$
(u+v)^{4} \geq 0
$$




$$
\begin{aligned}
(u, u)^{2}+4(u, u)(u, v) & +6(u, u)(v, v)+4(u, v)(v, v)+(v, v)^{2} \geq 0 \\
\left\{(u, u)^{2}+(v, v)^{2}+6(u, u)(v, v)\right\} & \geq-4(u, v)\{(u, u)+(v, v)\} \\
-\left\{(u, u)^{2}+(v, v)^{2}+6(u, u)(v, v)\right\} & \leq 4(u, v)\{(u, u)+(v, v)\} \\
\left\{(u, u)^{2}+(v, v)^{2}+6(u, u)(v, v)-4(u, u)(u, v)-4(u, v)(v, v)\right\} & =8(u, v)\{(u, u)+(v, v)\} \\
-(u-v)^{4} & =8(u, v)\{(u, u)+(v, v)\} \\
\|-(u-v)\|^{4} & =\|8(u, v)\{(u, u)+(v, v)\}\| \\
\|u-v\|^{4} & \leq 8\|u\|\|v\|\left\{\|u\|^{2}+\|v\|^{2}\right\}
\end{aligned}
$$

Also, we see that:

$$
\begin{aligned}
(u-v)^{4} & \geq 0 \\
(u, u)^{2}-4(u, u)(u, v) & +6(u, u)(v, v)-4(u, v)(v, v)+(v, v)^{2} \geq 0 \\
-4(u, v)\{(u, u)+(v, v)\} & =-\left\{(u, u)^{2}+(v, v)^{2}+6(u, u)(v, v)\right\} \\
4(u, v)\{(u, u)+(v, v)\} & \leq\left\{(u, u)^{2}+(v, v)^{2}+6(u, u)(v, v)\right\} \\
8(u, v)\{(u, u)+(v, v)\} & =(u+v)^{4} \\
\|8(u, v)\{(u, u)+(v, v)\}\| & =\|(u+v)\|^{4} \\
8\|u\|\|v\|\left\{\|u\|^{2}+\|v\|^{2}\right\} & \leq\|u+v\|^{4}
\end{aligned}
$$

We see from (4) and (5) that:

$$
\begin{aligned}
\|u-v\|^{4} & \leq\|u+v\|^{4} \\
\left(\|u-v\|^{4}\right)^{\frac{1}{4}} & =\left(\|u+v\|^{4}\right)^{\frac{1}{4}} \\
\Rightarrow\|u-v\| & =\|u+v\| \\
\Rightarrow\|u-v\| & \leq\|u\|+\|v\|
\end{aligned}
$$

Again, when $n=6$, we observe the following:

$$
\begin{aligned}
& \Rightarrow \quad(u+v)^{6} \geq 0 \\
& \Rightarrow \quad(u, u)^{3}+6(u, u)^{2}(u, v)+15(u, u)^{2}(v, v)+20(u, v)(u, u)(v, v) \\
& +\quad 15(u, u)(v, v)^{2}+6(u, v)(v, v)^{2}+(v, v)^{3} \geq 0 \\
& \Rightarrow \quad 6(u, v)\left\{(u, u)^{2}+(v, v)^{2}+\frac{20}{6}(u, u)(v, v)\right\} \geq-\left\{(u, u)^{3}+15(u, u)^{2}(v, v)\right. \\
& \left.+\quad 15(u, u)(v, v)^{2}+(v, v)^{3}\right\} \\
& \Rightarrow \quad-\left\{(u, u)^{3}+15(u, u)^{2}(v, v)+15(u, u)(v, v)^{2}+(v, v)^{3}\right\} \leq 6(u, v)\left\{(u, u)^{2}+(v, v)^{2}+\frac{20}{6}(u, u)(v, v)\right\} \\
& \Rightarrow \quad-(u-v)^{6}=12(u, v)\left\{(u, u)^{2}+(v, v)^{2}+\frac{20}{6}(u, u)(v, v)\right\} \\
& \Rightarrow \quad\left\|-(u-v)^{6}\right\|=\left\|12(u, v)\left\{(u, u)^{2}+(v, v)^{2}+\frac{20}{6}(u, u)(v, v)\right\}\right\| \\
& \Rightarrow \quad\|(u-v)\|^{6} \leq 12\|u\|\|v\|\left\{\|u\|^{4}+\|v\|^{4}+\frac{20}{6}\|u\|^{2}\|v\|^{2}\right\} .
\end{aligned}
$$


Also, we observe that:

$$
\begin{aligned}
& (u-v)^{6} \geq 0 \\
\Rightarrow & (u, u)^{3}-6(u, u)^{2}(u, v)+15(u, u)^{2}(v, v)-20(u, v)(u, u)(v, v) \\
+ & 15(u, u)(v, v)^{2}-6(u, v)(v, v)^{2}+(v, v)^{3} \geq 0 \\
\Rightarrow & -6(u, v)\left\{(u, u)^{2}+(v, v)^{2}+\frac{20}{6}(u, u)(v, v)\right\}=-\left\{(u, u)^{3}+15(u, u)^{2}(v, v)\right. \\
+ & \left.15(u, u)(v, v)^{2}+(v, v)^{3}\right\} \\
\Rightarrow & 6(u, v)\left\{(u, u)^{2}+(v, v)^{2}+\frac{20}{6}(u, u)(v, v)\right\} \leq\left\{(u, u)^{3}+15(u, u)^{2}(v, v)\right. \\
+ & \left.15(u, u)(v, v)^{2}+(v, v)^{3}\right\} \\
\Rightarrow & 12(u, v)\left\{(u, u)^{2}+(v, v)^{2}+\frac{20}{6}(u, u)(v, v)\right\}=(u+v)^{6} \\
\Rightarrow & \left\|12(u, v)\left\{(u, u)^{2}+(v, v)^{2}+\frac{20}{6}(u, u)(v, v)\right\}\right\|=\left\|(u+v)^{6}\right\| \\
\Rightarrow & 12\|u\|\|v\|\left\{\|u\|^{4}+\|v\|^{4}+\frac{20}{6}\|u\|^{2}\|v\|^{2}\right\} \leq\|(u+v)\|^{6}
\end{aligned}
$$

We see from inequalities (6) and (7) that:

$$
\begin{aligned}
\|u-v\|^{6} & \leq\|u+v\|^{6} \\
\left(\|u-v\|^{6}\right)^{\frac{1}{6}} & =\left(\|u+v\|^{6}\right)^{\frac{1}{6}} \\
\Rightarrow\|u-v\| & =\|u+v\| \\
\Rightarrow\|u-v\| & \leq\|u\|+\|v\|
\end{aligned}
$$

For any even positive integer $n$, we observe the following binomial inequality:

$$
\begin{aligned}
& (u+v)^{n} \geq 0 \\
\Rightarrow & (u, u)^{\frac{n}{2}}+{ }^{n} C_{1}(u, v)(u, u)^{\frac{n-2}{2}}+{ }^{n} C_{2}(u, u)^{\frac{n-2}{2}}(v, v)+{ }^{n} C_{3}(u, u)^{\frac{n-4}{2}}(v, v)(u, v) \\
+ & { }^{n} C_{4}(u, u)^{\frac{n-4}{2}}(v, v)^{2}+{ }^{n} C_{5}(u, u)^{\frac{n-6}{2}}(v, v)^{2}(u, v)+{ }^{n} C_{6}(u, u)^{\frac{n-6}{2}}(v, v)^{3} \\
+ & { }^{n} C_{7}(u, u)^{\frac{n-8}{2}}(v, v)^{3}(u, v)+\ldots+(u, u)^{\frac{n}{2}} \geq 0 \\
\Rightarrow & -n(u, v)\left\{(u, u)^{\frac{n-2}{2}}+\frac{1}{3 !}(n-1)(n-2)(u, u)^{\frac{n-4}{2}}(v, v)\right. \\
+ & \frac{1}{5 !}(n-1)(n-2)(n-3)(n-4)(u, u)^{\frac{n-6}{2}}(v, v)^{2} \\
+ & \left.\frac{1}{7 !}(n-1)(n-2)(n-3)(n-4)(n-5)(n-6)(u, u)^{\frac{n-8}{2}}(v, v)^{3}+\ldots+(v, v)^{\frac{n-2}{2}}\right\} \leq \\
& \left\{(u, u)^{\frac{n}{2}}+{ }^{n} C_{2}(u, u)^{\frac{n-2}{2}}(v, v)+{ }^{n} C_{2}(u, u)^{\frac{n-4}{2}}(v, v)^{2}+{ }^{n} C_{2}(u, u)^{\frac{n-6}{2}}(v, v)^{3}+\ldots+(v, v)^{\frac{n}{2}}\right\} \\
\Rightarrow & -\left\{(u, u)^{\frac{n}{2}}+{ }^{n} C_{2}(u, u)^{\frac{n-2}{2}}(v, v)+{ }^{n} C_{2}(u, u)^{\frac{n-4}{2}}(v, v)^{2}\right. \\
+ & \left.{ }^{n} C_{2}(u, u)^{\frac{n-6}{2}}(v, v)^{3}+\ldots+(v, v)^{\frac{n}{2}}\right\}
\end{aligned}
$$




$$
\begin{aligned}
& \leq n(u, v)\left\{(u, u)^{\frac{n-2}{2}}+\frac{1}{3 !}(n-1)(n-2)(u, u)^{\frac{n-4}{2}}(v, v)\right. \\
& +\frac{1}{5 !}(n-1)(n-2)(n-3)(n-4)(u, u)^{\frac{n-6}{2}}(v, v)^{2} \\
& \left.+\quad \frac{1}{7 !}(n-1)(n-2)(n-3)(n-4)(n-5)(n-6)(u, u)^{\frac{n-8}{2}}(v, v)^{3}+\ldots+(v, v)^{\frac{n-2}{2}}\right\} \\
& \Rightarrow \quad-(u-v)^{n}=n(u, v)\left\{(u, u)^{\frac{n-2}{2}}+\frac{1}{3 !}(n-1)(n-2)(u, u)^{\frac{n-4}{2}}(v, v)\right. \\
& +\frac{1}{5 !}(n-1)(n-2)(n-3)(n-4)(u, u)^{\frac{n-6}{2}}(v, v)^{2} \\
& \left.+\frac{1}{7 !}(n-1)(n-2)(n-3)(n-4)(n-5)(n-6)(u, u)^{\frac{n-8}{2}}(v, v)^{3}+\ldots+(v, v)^{\frac{n-2}{2}}\right\} \\
& \Rightarrow \quad\left\|-(u-v)^{n}\right\|=\| 2 n(u, v)\left\{(u, u)^{\frac{n-2}{2}}+\frac{1}{3 !}(n-1)(n-2)(u, u)^{\frac{n-4}{2}}(v, v)\right. \\
& +\frac{1}{5 !}(n-1)(n-2)(n-3)(n-4)(u, u)^{\frac{n-6}{2}}(v, v)^{2} \\
& \left.+\frac{1}{7 !}(n-1)(n-2)(n-3)(n-4)(n-5)(n-6)(u, u)^{\frac{n-8}{2}}(v, v)^{3}+\ldots+(v, v)^{\frac{n-2}{2}}\right\} \| \\
& \Rightarrow \quad\|(u-v)\| \leq 2 n\|u\| v \|\left\{(u, u)^{\frac{n-2}{2}}+\frac{1}{3 !}(n-1)(n-2)(u, u)^{\frac{n-4}{2}}(v, v)\right. \\
& +\frac{1}{5 !}(n-1)(n-2)(n-3)(n-4)(u, u)^{\frac{n-6}{2}}(v, v)^{2} \\
& \left.+\frac{1}{7 !}(n-1)(n-2)(n-3)(n-4)(n-5)(n-6)(u, u)^{\frac{n-8}{2}}(v, v)^{3}+\ldots+(v, v)^{\frac{n-2}{2}}\right\} \|
\end{aligned}
$$

On the other hand, we see that:

$$
\begin{aligned}
& (u-v)^{n} \geq 0 \\
\Rightarrow & (u, u)^{\frac{n}{2}}-{ }^{n} C_{1}(u, v)(u, u)^{\frac{n-2}{2}}+{ }^{n} C_{2}(u, u)^{\frac{n-2}{2}}(v, v)-{ }^{n} C_{3}(u, u)^{\frac{n-4}{2}}(v, v)(u, v)+{ }^{n} C_{4}(u, u)^{\frac{n-4}{2}}(v, v)^{2} \\
- & { }^{n} C_{5}(u, u)^{\frac{n-6}{2}}(v, v)^{2}(u, v)+{ }^{n} C_{6}(u, u)^{\frac{n-6}{2}}(v, v)^{3}-{ }^{n} C_{7}(u, u)^{\frac{n-8}{2}}(v, v)^{3}(u, v)+\ldots+(u, u)^{\frac{n}{2}} \geq 0 \\
\Rightarrow & -n(u, v)\left\{(u, u)^{\frac{n-2}{2}}+\frac{1}{3 !}(n-1)(n-2)(u, u)^{\frac{n-4}{2}}(v, v)\right. \\
+ & \frac{1}{5 !}(n-1)(n-2)(n-3)(n-4)(u, u)^{\frac{n-6}{2}}(v, v)^{2} \\
+ & \left.\frac{1}{7 !}(n-1)(n-2)(n-3)(n-4)(n-5)(n-6)(u, u)^{\frac{n-8}{2}}(v, v)^{3}+\ldots+(v, v)^{\frac{n-2}{2}}\right\}= \\
- & -\left\{(u, u)^{\frac{n}{2}}+{ }^{n} C_{2}(u, u)^{\frac{n-2}{2}}(v, v)+{ }^{n} C_{2}(u, u)^{\frac{n-4}{2}}(v, v)^{2}+{ }^{n} C_{2}(u, u)^{\frac{n-6}{2}}(v, v)^{3}+\ldots+(v, v)^{\frac{n}{2}}\right\} \\
\Rightarrow & n(u, v)\left\{(u, u)^{\frac{n-2}{2}}+\frac{1}{3 !}(n-1)(n-2)(u, u)^{\frac{n-4}{2}}(v, v)^{\frac{n}{2}}(v, v)^{2}\right. \\
+ & \frac{1}{5 !}(n-1)(n-2)(n-3)(n-4)(u, u)^{\frac{n-6}{2}} \\
+ & \left.\frac{1}{7 !}(n-1)(n-2)(n-3)(n-4)(n-5)(n-6)(u, u)^{\frac{n-8}{2}}(v, v)^{3}+\ldots+(v, v)^{\frac{n-2}{2}}\right\} \\
\leq & \left\{(u, u)^{\frac{n}{2}}+{ }^{n} C_{2}(u, u)^{\frac{n-2}{2}}(v, v)+{ }^{n} C_{2}(u, u)^{\frac{n-4}{2}}(v, v)^{2}+{ }^{n} C_{2}(u, u)^{\frac{n-6}{2}}(v, v)^{3}+\ldots+(v, v)^{\frac{n}{2}}\right\}
\end{aligned}
$$




$$
\begin{aligned}
& \Rightarrow 2 n(u, v)\left\{(u, u)^{\frac{n-2}{2}}+\frac{1}{3 !}(n-1)(n-2)(u, u)^{\frac{n-4}{2}}(v, v)\right. \\
& +\frac{1}{5 !}(n-1)(n-2)(n-3)(n-4)(u, u)^{\frac{n-6}{2}}(v, v)^{2} \\
& \left.+\frac{1}{7 !}(n-1)(n-2)(n-3)(n-4)(n-5)(n-6)(u, u)^{\frac{n-8}{2}}(v, v)^{3}+\ldots+(v, v)^{\frac{n-2}{2}}\right\} \\
& =(u+v)^{n} \\
& \Rightarrow \quad \| 2 n(u, v)\left\{(u, u)^{\frac{n-2}{2}}+\frac{1}{3 !}(n-1)(n-2)(u, u)^{\frac{n-4}{2}}(v, v)\right. \\
& +\frac{1}{5 !}(n-1)(n-2)(n-3)(n-4)(u, u)^{\frac{n-6}{2}}(v, v)^{2} \\
& \left.+\frac{1}{7 !}(n-1)(n-2)(n-3)(n-4)(n-5)(n-6)(u, u)^{\frac{n-8}{2}}(v, v)^{3}+\ldots+(v, v)^{\frac{n-2}{2}}\right\} \| \\
& =\left\|(u+v)^{n}\right\| \\
& \Rightarrow 2 n\|u\|\|v\| \|\left\{(u, u)^{\frac{n-2}{2}}+\frac{1}{3 !}(n-1)(n-2)(u \cdot u)^{\frac{n-4}{2}}(v, v)\right. \\
& +\frac{1}{5 !}(n-1)(n-2)(n-3)(n-4)(u, u)^{\frac{n-6}{2}}(v, v)^{2} \\
& \left.+\frac{1}{7 !}(n-1)(n-2)(n-3)(n-4)(n-5)(n-6)(u, u)^{\frac{n-8}{2}}(v, v)^{3}+\ldots+(v, v)^{\frac{n-2}{2}}\right\} \| \\
& \leq\|(u+v)\|^{n}
\end{aligned}
$$

By transitivity, inequalities (8) and (9) yields

$$
\begin{aligned}
\|u-v\|^{n} & \leq\|u+v\|^{n} \\
\left(\|u-v\|^{n}\right)^{\frac{1}{n}} & =\left(\|u+v\|^{n}\right)^{\frac{1}{n}} \\
\Rightarrow\|u-v\| & =\|u+v\| \\
\Rightarrow\|u-v\| & \leq\|u\|+\|v\|
\end{aligned}
$$

\subsection{The Proof of the Triangle Inequality through Euclidean norm}

In this subsection, we will establish the same result as in (10), by making use of both the binomial inequality of two vector points and the Young's inequality.

Definition 2 (Young's Inequality). Let $1<p<\infty, q$ the conjugate of $p$, and any two vectors $u$ and $v$, then

$$
(u, v) \leq \frac{u^{p}}{p}+\frac{v^{q}}{q}
$$

where

$$
\frac{1}{p}+\frac{1}{q}=1
$$

see [14]. 
Firstly, we observe the two vectors in the Hilbert space as:

$$
\begin{aligned}
(u+v)^{2} & \geq 0 \\
(u, u)+2(u, v)+(v, v) & \geq 0 \\
-\{(u, u)+(v, v)\} & \leq 2(u, v) \\
-\{(u, u)+(v, v)-2(u, v)\} & \leq 2(u, v) \\
-(u-v)^{2} & =2(u, v) \\
\left\|-(u-v)^{2}\right\| & =\|2(u, v)\| \\
\|(u-v)\|^{2} & =|2||(u, v)| \\
\Rightarrow\|(u-v)\|^{2} & \leq 2|(u, v)|
\end{aligned}
$$

Applying the Young's inequality of two vectors

$$
\begin{aligned}
|(u, v)| & \leq\left|\frac{u^{2}}{2}+\frac{v^{2}}{2}\right| \\
\|(u, v)\| & \leq \frac{|u|^{2}}{2}+\frac{|v|^{2}}{2},
\end{aligned}
$$

where $p=q=2$. Substituting the inequality in (12) into left hand side of inequality (11) yields:

$$
\begin{aligned}
\|(u-v)\|^{2} & \leq 2\left\{\frac{|u|^{2}}{2}+\frac{|v|^{2}}{2}\right\} \\
\Rightarrow\|(u-v)\|^{2} & =|u|^{2}+|v|^{2} \\
\Rightarrow\|(u-v)\| & \leq \sqrt{|u|^{2}+|v|^{2}} .
\end{aligned}
$$

On the other hand, the norm of sum of two vectors in Hilbert space was observed as:

$$
\begin{aligned}
(u+v)^{2} & \geq 0 \\
\Rightarrow-\{(u, u)+(v, v)\} & \leq 2(u, v) \\
\Rightarrow-2(u, v) & \leq\{(u, u)+(v, v)+2(u, v)\} \\
\Rightarrow-2(u, v) & =(u+v)^{2} \\
\Rightarrow\|-2(u, v)\| & =\left\|(u+v)^{2}\right\| \\
\Rightarrow 2|(u, v)| & \leq\|u+v\|^{2} \\
\Rightarrow 2\left\{\frac{|u|^{2}}{2}+\frac{|v|^{2}}{2}\right\} & \leq\|u+v\|^{2} \\
\Rightarrow|u|^{2}+|v|^{2} & =\|u+v\|^{2} \\
\Rightarrow \sqrt{|u|^{2}+|v|^{2}} & \leq\|u+v\|^{2}
\end{aligned}
$$

By transitivity, the inequalities (13) and (14) yields

$\|u-v\| \leq\|u\|+\|v\|$ 


\section{Discussion}

We have obtained the generalized alternative way of proving the triangle inequality in (1) for any positive integer $n$, unlike the results obtained by other researchers. Also, the second alternative proof of the triangle inequality establishes 2- norm of any two vector points in the Hilbert space.

\section{Conclusion}

We have shown the general alternative ways of proving the triangle inequality $\|u-v\| \leq$ $\|u\|+\|v\|$, through the binomial inequality and also, through the Euclidean norm in Hilbert space.

\section{References}

[1] C. L. Fleming, S. E. Griffis and J. E. Bell, The effects of triangle inequality on the vehicle routing problem, European Journal of Operational Research 224, 1 , (2013).pp. 1-7

[2] B. Kr. Patra, International conference on communication computing and security: Using the triangle inequality to accelerate density based outlier detection method, Procedia Technology 6 , (2012).pp. 469-474

[3] A. M. Fink, An essay on the history of inequalities, Journal of Mathematical Analysis and Applications, 249 , (2000).pp. 118-134.

[4] H. P. Mulholland, On generalizations of Minkowski's inequality in the form of a triangle inequality, Proceedings of the London mathematical society vol. 2-51, no. 1, (1949). pp. 294-307.

[5] K-S. Saito, R. An, H. Mizuguchi and K-I. Mitani, Another aspect of triangle inequality, International scholarly research network(2011). pp. 5 doi : 10.5402/2011/514184.

[6] K-I. Mitani and K-S Saito, On sharp triangle inequalities in Banach spaces II, Journal of inequalities and applications, (2010). pp. 17 doi : 10.1155/2010/323609

[7] S. Saitoh, Generalizations of the triangle inequality, Journal of Inequalities in Pure and Applied Mathematics vol. 4, no. 3, (2003)

[8] T. Izumida, K-I. Mitani and K-S Saito, Another approach to characterization of generalized triangle inequality in normed space, Central European Journal of Mathematics 12, $11,(2014)$.pp. 1615-1623

[9] F. Dadipour, M.S. Moslehian, J. M. Rassias and S-E. Takahasi, Characterization of a generalized triangle inequality in normed spaces, Nonlinear Analysis vol. 75, no. 2, (2012). pp. 735-741 
[10] J. T. Scheick, Linear Algebra with Applications, Prentice-Hall, Inc, New York; 1997.

[11] N. Minculete and R. Pãltãnea, Improved estimates for the triangle inequality, Journal of Inequality and Applications , (2017). doi. 10.1186/s13660 - $016-1281-z$

[12] J. T. Oden, Applied function analysis; a first course for students of mechanics and engineering science, Englewood Cliffs, N.J. Prentice-Hall, 1979.

[13] B. Kolman and D. R. Hill, Elementary Linear Algebra, Prentice-Hall, Inc, New Jersey; 2000 .

[14] H. Royden and P. Fitxpatrick, Real Analysis. Pearson Education, Inc, $4^{\text {th }}$ ed., Upper saddle River; 2010. 